\title{
Prognostic Utility of Dobutamine Stress Echocardiography in the Obese African American Population
}

\author{
Arfaat Khan, Eduardo Valdiviezo, Gurjit Singh, Somsupha Kanjanauthai, Waheeda Nazneen, \\ Karthik Ananthasubramaniam ${ }^{\#}$ \\ Heart and Vascular Institute, The Department of Internal Medicine, Henry Ford Hospital, Detroit, USA \\ Email: "kananth1@hfhs.org
}

Received January 22, 2012; revised April 21, 2012; accepted April 30, 2012

\begin{abstract}
Background: The predictive value of Dobutamine Stress Echocardiography (DSE) in the obese African American patient population is not well known. Methods: We conducted a retrospective study of 555 African American patients between 1/1/2001-12/31/2001. DSE responses were classified into normal, ischemia, scar, or scar $+/-$ ischemia. Endpoints utilized were all cause death (ACD) and major adverse cardiac events (MACE). Results: There were a total of 409 obese patients and 146 non-obese patients. By multi-variate analysis only the scar group in the obese population predicted ACD $(\mathrm{p}=0.003)$ and combined MACE $(\mathrm{p}=0.014)$. Kaplan Meir analysis demonstrated that only the scar group was associated with decrease in freedom from all cause death $(\mathrm{p}<0.001)$ and combined MACE $(\mathrm{p}<0.001)$. Conclusion: DSE retains its prognostic power in the obese African American population and scar has important implications for MACE.
\end{abstract}

Keywords: African American; Obesity; Stress Echocardiography; Dobutamine Stress Echocardiography; Outcomes

\section{Introduction}

Dobutamine Stress Echocardiography (DSE), with or without adjunctive atropine, is a widely accepted and useful noninvasive test for the diagnosis, risk stratification and prognosis of coronary artery disease (CAD) [1-4]. DSE has also been shown to provide important prognostic information in men and women [5] as well as in special subsets such as diabetics [6]. However, the prognostic utility of DSE in certain patient populations such as African Americans to evaluate CAD and predict cardiac events is scarce. Epidemiological and randomized cardiovascular research trials have demonstrated an under representation of the African American population ranging from only $2 \%$ - $9 \%$ in their study population [7]. Our group and others have previously shown using stress echocardiography that African Americans have a higher risk of cardiac events as compared to Caucasians [8]. Prior data have shown that nuclear perfusion in African Americans with normal or low risk scans have higher rates of cardiac events as compared to Caucasians with normal or low risk scans [9-11]. The higher mortality in

\footnotetext{
*Disclosures: KA receives Research grant support: Astellas Pharma Global development Inc, GE healthcare and is on Speakers Bureau for Astellas Pharma Global development Inc, Lantheus Medical Imaging and has received consulting fees and travel support from Lantheus Medical Imaging.

${ }^{\#}$ Corresponding author.
}

African Americans may be attributed to a higher prevalence of individual risk factors such as hypertension and type II diabetes mellitus [9]. There is also a higher prevalence of obesity in the African American population [7]. Obesity (body mass index (BMI) $>30$ ) has long been associated with an increase risk for CAD [12-14]. The higher incidence of CAD may be due to associated risk factors such as diabetes and hypertension, similar to the African American population. However, studies have found that obesity is an independent cardiovascular risk factor even when adjusting for associated risk factors $[15,16]$. The American Heart Association has identified obesity as an independent risk factor for coronary heart disease [12]. Even after adjusting for these risk factors, cardiovascular mortality remains high in the African American population.

Although there is a higher prevalence of obesity in African Americans, the prognostic utility of DSE in obese African Americans to evaluate $\mathrm{CAD}$ and predict cardiac events is scarce. In addition, performance of DSE in obese patients as compared to lean patients is a challenge given suboptimal image quality leading to poor endocardial definition and thus underestimation of ischemia. In a study by Hu et al., in 62 overweight or obese patients there was significant improvement in sensitivity, specificity and overall accuracy of DSE enhanced with echocardiographic contrast agent when compared to non- 
contrast DSE using coronary angiography as gold standard [17]. The purpose of our study was to determine whether DSE is a robust non-invasive modality to evaluate $\mathrm{CAD}$ and predict cardiac events in the obese African American population.

\section{Methods}

\subsection{Patient Population}

A retrospective chart review was performed of patients referred to our institution for DSE between January-December 2001. This consisted of a total of 555 African American patients. Baseline demographics and clinical risk factors were collected. Obese patients were identified based on $\mathrm{BMI} \geq 30$. Of 555 patients, $74 \%$ were classified as obese. Indications for the DSE in the obese patients were as follows: $67 \%$ for chest pain, $10 \%$ for shortness of breath, $8 \%$ for routine pre-operative risk assessment and $15 \%$ for other reasons which were not specified.

\subsection{DSE Protocol}

Baseline images were obtained in the standard parasternal and apical 4, 3 and 2 chamber views. An infusion of dobutamine was started at $10 \mu \mathrm{g} / \mathrm{kg} / \mathrm{min}$ and increased every $3 \mathrm{~min}$ to $20,30,40 \mu \mathrm{g} / \mathrm{kg} / \mathrm{min}$ until the target heart rate was achieved. If the heart rate was less than $100 \mathrm{bpm}$ at $20 \mu \mathrm{g}$, atropine in $0.25 \mathrm{mg}$ doses up to a total dose of 2 mg was given till $85 \%$ predicted maximal heart rate was achieved. Dobutamine infusion was stopped if the target heart rate was achieved, protocol was completed, or for standard indications such as severe chest pain, $>2 \mathrm{~mm} \mathrm{ST}$ depression, marked hypertensive response of $>210 \mathrm{~mm}$ hg systolic blood pressure, development of new regional wall motion abnormalities or if requested by patient. Images were digitized and displayed in 4 quadrant views for side by side comparison of baseline, $20 \mu \mathrm{g}, 30 \mu \mathrm{g}$, and peak images. All studies were performed using second harmonic imaging. Echocardiographic contrast agents for left ventricular opacification were used when $>2$ contiguous segments based on a 16 segment model was not optimally visualized.

\subsection{Echocardiography Analysis}

Echocardiographic responses to stress were defined as normal if the left ventricle had normal function at rest with improved function during stress, ischemia if the left ventricle developed a new wall motion abnormality or worsening of resting hypokinesia with stress, and as scar if there was a wall motion abnormality at rest without development of ischemia. Results were classified into normal, ischemia, scar, or scar + ischemia. All images were reviewed by experienced echocardiographers with
$>5$ years experience in stress echocardiography. The stress echo results were classified based on initial interpretation. The American Society of Echocardiography based 16 segment model was used for visual wall motion analysis (need to insert reference). Ejection fraction (EF) $>/=50 \%$ was considered normal. Ejection fraction calculation was performed by visual estimation by the reading echocardiographer. DSE electrocardiographic analysis was done by the reading echocardiographer in conjunction with the echo analysis. Ischemic response on the electrocardiogram was considered when there was $>1$ $\mathrm{mm}$ ST segment deviation from baseline in two or more contiguous leads during dobutamine infusion or during recovery. Per our lab protocol the overall results of the DSE was considered not consistent with ischemia if electrocardiographic changes for ischemia were not corroborated by echo abnormalities unless it was felt that echocardiographic images were not conclusive or optimal.

\subsection{Follow Up and End-Points}

Follow up was obtained by review of hospital electronic medical records. Median follow up was 72 months. The end points assessed were all cause mortality and MACE; MACE included all cause death and non-fatal MI. Myocardial infarction was defined as symptoms of myocardial ischemia accompanied by increase in markers of myocardial necrosis.

\subsection{Statistical Analysis}

Sensitivity, specificity, positive predictive value, and negative predictive value of DSE for MACE prediction were calculated. If one patient had more than one MACE during the follow up period, he or she was treated as an "event patient" and accounted for as one event. The time to event in these patients was the time from DSE to the first qualifying MACE. Univariate analysis of all variables was performed using the chi-square and Student $\mathrm{T}$ tests. Cox proportional hazards regression analysis was performed to identify multivariate predictors of MACE post DSE. All of the variables on the univariate analysis rendering a log-rank $p$ value of 0.1 or less were included in the multivariate analysis. Survival free of the end point of interest was estimated by the Kaplan-Meier method.

\section{Results}

\subsection{Baseline Characteristics}

Mean age in the obese group $(\mathrm{N}=409)$ was $64 \pm 11.9$ years and in the non-obese group $(\mathrm{N}=146)$ was $74 \pm 12$ years. In the obese and non-obese group $283(70 \%)$ and $90(62 \%)$ were females respectively. Other clinical and demographic characteristics are summarized in Table $\mathbf{1}$ Multivariate analysis of baseline clinical variables which 
Table 1. Baseline characteristics and hemodynamic and electrocardiographic features of the 2 groups.

\begin{tabular}{|c|c|c|c|}
\hline Variable & Non-Obese $(\mathrm{N}=146)$ & Obese $(\mathrm{N}=409)$ & p-value \\
\hline Age in Years & $73.7 \pm 12.0$ & $63.9 \pm 11.9$ & $<0.001(\mathrm{~T})^{*}$ \\
\hline Female & $90 / 146(61.6 \%)$ & $283 / 407(69.5 \%)$ & $0.081(\mathrm{C})$ \\
\hline $\mathrm{DM}$ & $59 / 146(40.4 \%)$ & $203 / 409(49.6 \%)$ & $0.055(\mathrm{C})$ \\
\hline Known CAD & $50 / 146(34.2 \%)$ & $93 / 408(22.8 \%)$ & $0.007(\mathrm{C})^{*}$ \\
\hline Prior MI & $29 / 146(19.9 \%)$ & $86 / 408(21.1 \%)$ & $0.756(\mathrm{C})$ \\
\hline Previous Revascularization & $34 / 146(23.3 \%)$ & $61 / 408(15.0 \%)$ & $0.022(\mathrm{C})^{*}$ \\
\hline $\mathrm{EF}<50$ & $29 / 146(19.9 \%)$ & $51 / 408(12.5 \%)$ & $0.030(\mathrm{C})^{*}$ \\
\hline HTN & $135 / 146(92.5 \%)$ & $393 / 409(96.1 \%)$ & $0.081(\mathrm{C})$ \\
\hline GFR $<60$ & $54 / 146(37.0 \%)$ & $103 / 409(25.2 \%)$ & $0.007(\mathrm{C})^{*}$ \\
\hline Hypotensive Response & $38 / 144(26.4 \%)$ & 70/394 (17.8\%) & $0.027(\mathrm{C})^{*}$ \\
\hline Hypertensive Response & $19 / 144(13.2 \%)$ & 46/394 (11.7\%) & $0.632(\mathrm{C})$ \\
\hline Submaximal Stress & $51 / 145(35.2 \%)$ & $112 / 406(27.6 \%)$ & $0.086(\mathrm{C})$ \\
\hline Ischemic ECG & $6 / 146(4.1 \%)$ & $29 / 408(7.1 \%)$ & $0.201(\mathrm{C})$ \\
\hline Baseline ECG LVH & $15 / 146(10.3 \%)$ & $56 / 408(13.7 \%)$ & $0.284(\mathrm{C})$ \\
\hline Baseline LBBB & $0 / 146(0.0 \%)$ & $12 / 407(2.9 \%)$ & $0.042(\mathrm{~F})^{*}$ \\
\hline
\end{tabular}

Numeric data is given as mean \pm standard deviation. Categorical data is given as fraction (percent). $(\mathrm{T})=\mathrm{Student} 2$-sample $\mathrm{T}$-test; $(\mathrm{C})=\mathrm{Chi}$-square test; $(\mathrm{F})=$ Fisher exact test. $*$ Statistically significant, $\mathrm{p}<0.05$.

predicted obesity were female gender $(\mathrm{p}<0.03$, OR $1.669 ; 95 \%$ CI 1.04 - 2.67), DM ( $<0.004$, OR 1.98; $95 \%$ CI $1.24-3.15$ ), prior MI ( $<<0.001$, OR $5.0195 \%$ CI 1.92 - 13.05), and presence of hypertension ( $\mathrm{p}<0.01$, OR 3.38; 95\% CI 1.24 - 9.18).

\subsection{Obese Group vs Non-Obese Group}

The obese group in comparison to the non-obese group demonstrated no significant difference in freedom from all-cause death $(p=0.09)$ or MACE $(p>0.5)$.

\subsection{Obese Group}

Multivariate analysis of baseline characteristics revealed that only the female gender ( $p<0.02$, HR $3.186 ; 95 \%$ CI $1.1-8.8)$ predicted all cause death. Risk factors associated with the development of a non-fatal MI were prior history of MI ( $<<0.001$, HR 9.51; 95\% CI 2.7 - 33). Predictors for heart failure admission was $\mathrm{EF}<50 \%$ ( $\mathrm{p}=$ 0.002 , HR 3.98; 95\% CI 1.65 - 9.59), and for MACE was also $\mathrm{EF}<50 \%(\mathrm{p}=0.03$, HR 2.05; 95\% CI $1.05-3.99)$.

\subsection{DSE-Non Obese Group $(n=140)$}

One hundred and fourteen (81\%) patients had a normal DSE result. $8(5.7 \%)$ had an ischemic response. $13(9.3 \%)$ demonstrated presence of only scar. 5 (3.6\%) demon- strated presence of scar and ischemia.

\subsection{DSE-Obese Group $(n=404)$}

Three hundred and forty four (85.1\%) patients had a normal DSE result. $20(5 \%)$ had an ischemic response. $30(7.4 \%)$ demonstrated presence of only scar. $10(2.5 \%)$ demonstrated presence of scar and ischemia. Multivariable analysis demonstrated that only the scar group compared to the normal group predicted all-cause death (scar vs. normal $\mathrm{p}=0.003$, OR 5.97; 95\% CI $1.81-19.57$ ) and combined MACE (scar vs. normal $\mathrm{p}=0.014$, OR 2.85; 95\% CI 1.23 - 6.60) (See Table 2). Kaplan Meier analysis demonstrated that only the scar group compared to the normal group was associated with decrease in freedom from all cause death $(\mathrm{p}<0.001)$, and combined $\operatorname{MACE}(\mathrm{p}=0.001)$ (See Figures 1(a), (b)).

\subsection{Revascularization Post DSE}

Only 11 patients underwent revascularization by either percutaneous coronary intervention or by coronary artery bypass grafting after DSE within first 90 days after DSE. When we repeated the analysis by excluding these patients in the obese group, the group with scar alone was an independent predictor of all cause death ( $\mathrm{p} \leq 0.002$, HR 6.8, 955 CI 2.01 - 23.2) and combined MACE ( $\mathrm{p}=$ 0.033 , HR 2.3, 95\% CI 1.07 - 5.06) with previous MI (p 


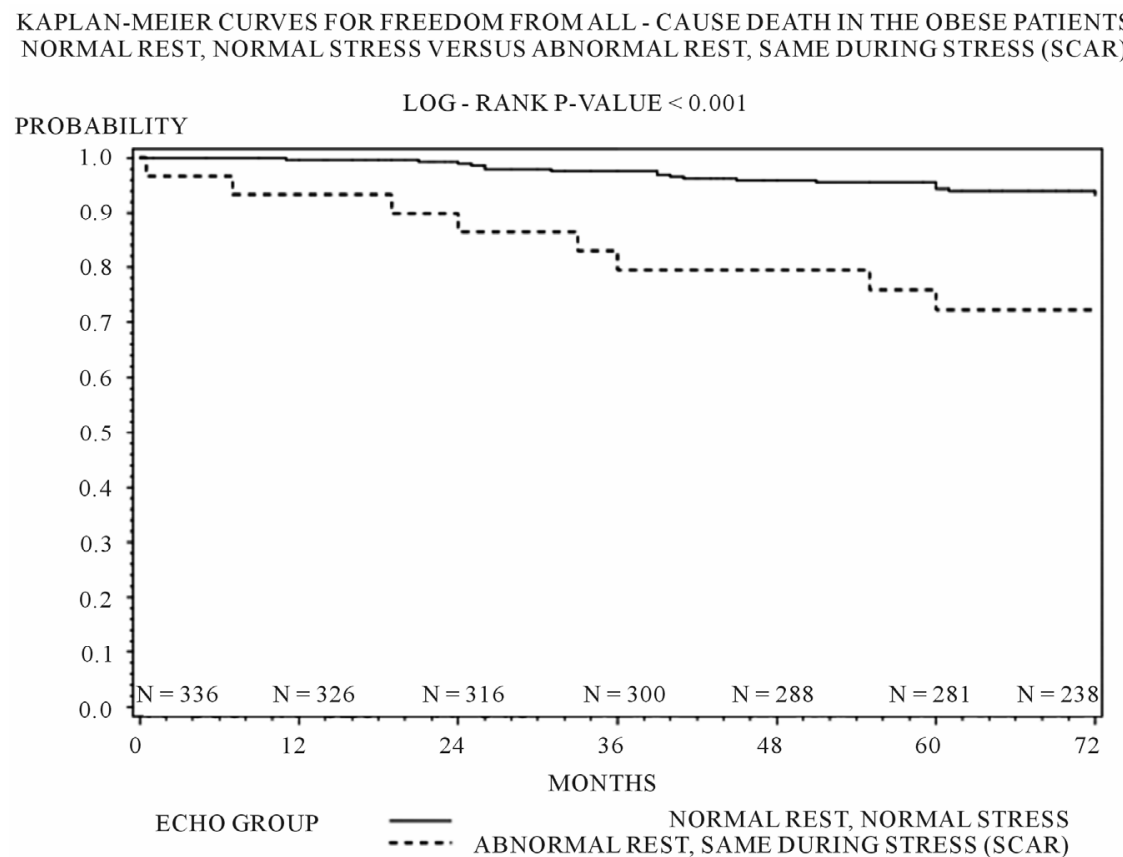

(a)

KAPLAN-MEIER CURVES FOR FREEDOM FROM ALL - CAUSE DEATH IN THE OBESE PATIENTS NORMAL REST, NORMAL STRESS VERSUS ABNORMAL REST, SAME DURING STRESS (SCAR)

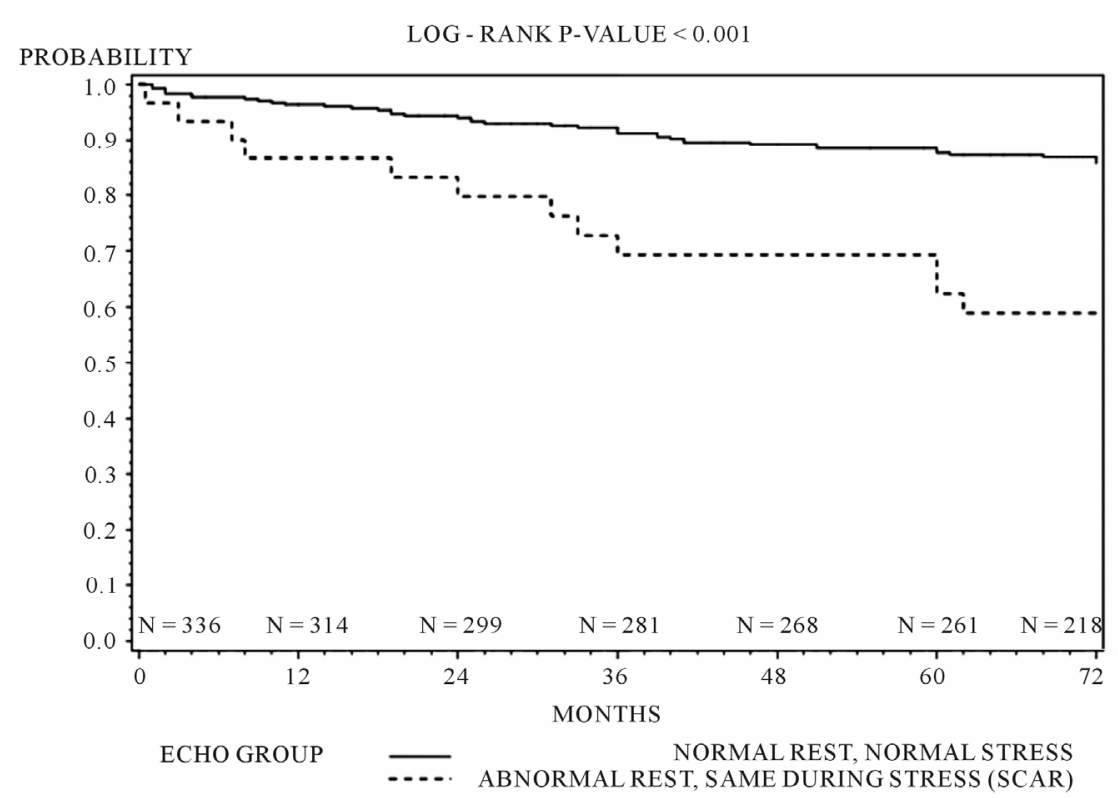

(b)

Figure 1. (a) Kaplan-Meier freedom from all cause death by echo groups in the obese patients (log rank p value $<0.001)$; (b) Kaplan-Meier freedom from combined MACE (all-cause death or non-fatal MI) in the obese patients (log rank p value $<$ 0.001).

Table 2. Multivariate predictors of all cause death and MACE.

\begin{tabular}{cccccc}
\hline Endpoint & Stress Test Result & p-value & Hazard Ratio & 95\% Hazard Ratio Confidence Limits \\
\hline All-cause death & Scar vs. Normal & 0.003 & 5.97 & 1.82 & 19.57 \\
Combined MACE & Scar vs. Normal & 0.014 & 2.85 & 1.23 & 6.60 \\
\hline
\end{tabular}


$=0.012)$ and $\mathrm{EF}<50 \%(\mathrm{p}=0.022)$ also being significant baseline clinical predictors of combined MACE.

\section{Discussion}

Our study demonstrates that when comparing an obese African American population to a non-obese African American population there was no difference in endpoints of all cause death or MACE based on results of DSE. However, amongst obese African Americans who were found to have presence of scar on DSE, there was a significantly increased risk of all-cause mortality. Presence of scar on DSE in obese African Americans predicted higher all-cause mortality and combined MACE (all cause death or non-fatal MI). Kaplan Meier analysis demonstrated that the obese Africans Americans with scar on DSE had decrease in freedom from combined MACE compared to patients with a normal DSE result. Thus essentially resting echocardiographic determination of wall motion plays an important role in the risk stratification of obese African Americans, as scar seems to be the major predictor of MACE.

There is scarcity of information on the usefulness of DSE in the obese African American population. Sawada and colleagues recently evaluated DSE in African Americans populations without designation of obesity status. They concluded that history of MI, reduced fractional shortening, increased left atrial dimension, and stress induced ischemia were predictors of MI and cardiac death [18]. Similarly, in our study, presence of prior MI was an independent predictor of development of non-fatal MI. However, the presence of ischemia alone with or without presence of scar was not predictive of all cause death or MACE. It is well known that low EF is a major driver of death compared to ischemia, which serves as a predictor of non-fatal MI. The majority of our study patients $(80 \%)$ had a normal EF. Thus, patients with ischemia but preserved ejection fraction have low mortality overall. Furthermore, since burden of ischemia is a major driver of events, patients with mild wall motion abnormalities or single vessel ischemia on medical therapy largely have benign outcomes, which may explain the lack of predictive value of ischemia for MACE and death in our study. Finally, the presence of obesity may also have a possible role. Clear visualization of the left ventricular endocardium is necessary for assessment of a wall motion abnormality. In the obese population this at times may be difficult due to poor echocardiographic windows despite contrast use, which has been shown to increase the diagnostic value of DSE in detecting CAD [17]. The frequency of use of contrast echo in our patient population is not known. The incremental benefit of contrast echo in determining whether ischemia alone was predictive of cardiac events can not be made at this time.

Since routine coronary angiography was not performed in patients despite abnormal DSE it is quite possible that some of the "abnormal DSE" could have been a false positive and thus not be predictive of events.

The presence of scar in our study population was shown to have important implications for prediction and freedom from MACE. Utilization of either DSE for wall motion abnormalities or by cardiac magnetic resonance imaging by late gadolinium enhancement for presence of scar, have recognized that presence of scar with or with out ischemia is associated with approximately $4-11$ fold hazards increase risk for MACE and death [3,19-24]. Our study reconfirms this finding, demonstrating presence of scar by DSE carries an increase risk of all cause death and combined MACE for obese African Americans; similarly, presence of scar on DSE in the general population has been associated with adverse outcomes.

\section{Limitations}

The limitations of this study include that this was a retrospective study with its inherent limitations and bias. Patients did not undergo routine coronary angiography and thus the true sensitivity, specificity, positive and negative predictive values of DSE for detecting CAD in the obese population cannot be determined from this study. The use of contrast echo in our patient population was not consistent given its introduction to our lab at the time of this study and so it is also unknown whether or not this may have affected the results of DSE in prediction of MACE. Also wall motion scoring was not done in our lab at the time of the study. However, all interpreting echocardiographers were experienced with at least Level 2 and in many cases Level 3 equivalent training in interpretation of echocardiographic studies. Hence, we believe this is representative of real world DSE interpretation of most practices.

\section{Conclusion}

Our study shows that DSE remains a powerful diagnostic tool in the obese African American population. The presence of scar alone demonstrates an increase risk for prediction of all-cause death and combined MACE. This particular group of patients requires close monitoring and aggressive treatment of associated risk factors.

\section{REFERENCES}

[1] E. H. Steinberg, L. Madmon, C. P. Patel, S. P. Sedlis, I. Kronzon and J. L. Cohen. "Long-Term Prognostic Significance of Dobutamine Echocardiography in Patients with Suspected Coronary Artery Disease: Results of a 5-Year Follow-Up Study," Journal of the American College of Cardiology, Vol. 29, No. 5, 1997, pp. 969-973. 
doi:10.1016/S0735-1097(97)00032-6

[2] D. Poldermans, P. M. Fioretti, E. Boersma, J. H. Cornel, F. Borst, E. G. J. Vermeulen, M. Arnese, A. El-Hendy and J. R. T. C. Roelandt, "Dobutamine-Atropine Stress Echocardiography and Clinical Data for Predicting Late Cardiac Events in Patients with Suspected Coronary Artery Disease," The American Journal of Medicine, Vol. 97, No. 2, 1994, pp. 119-125. doi:10.1016/0002-9343(94)90021-3

[3] J. Krivokapich, J. S. Child, D. O. Walter and A. Garfinkel. "Prognostic Value of Dobutamine Stress Echocardiography in Predicting Cardiac Events in Patients with Known or Suspected Coronary Artery Disease," Journal of the American College Cardiology, Vol. 33, No. 3, 1999, pp. 708-716. doi:10.1016/S0735-1097(98)00632-9

[4] S. C. Chuah, P. A. Pellikka, V. L. Roger, R. B. McCully and J. B. Seward, "Role of Dobutamine Stress Echocardiography in Predicting Outcome in 860 Patients with Known or Suspected Coronary Artery Disease," Circulation, Vol. 97, 1998, pp. 1474-1480.

[5] L. J. Shaw, C. Vasey, S. Sawada, C. Rimmerman and T. H. Marwick, "Impact of Gender on Risk Stratification by Exercise and Dobutamine Stress Echocardiography: LongTerm Mortality in 4234 Women and 6898 Men," European Heart Journal, Vol. 26, No. 5, 2005, pp. 447-456. doi:10.1093/eurheartj/ehi102

[6] L. Cortigiani, R. Bigi, R. Sicari, P. Landi, F. Bovenzi and E. Picano, "Prognostic Value of Pharmacological Stress Echocardiography in Diabetic and Nondiabetic Patients with Known or Suspected Coronary Artery Disease," Journal of the American College of Cardiology, Vol. 47, No. 3, 2006, pp. 605-610. doi:10.1016/j.jacc.2005.09.035

[7] R. F. Gillum and K. C. Liu, "Coronary Heart Disease Mortality in United States Blacks, 1940-1978: Trends and Unanswered Questions," American Heart Journal, Vol. 108, No. 3, 1984, pp. 728-732. doi:10.1016/0002-8703(84)90665-3

[8] A. V. Srivastava, K. Ananthasubramaniam, S. J. Patel, N. Lingam and G. Jacobsen, "Prognostic Implications of Negative Dobutamine Stress Echocardiography in African Americans Compared to Caucasians," Cardiovascular Ultrasound, Vol. 6, No. 1, 2008, p. 20. doi:10.1186/1476-7120-6-20

[9] O. O. Akinboboye, O. Idris, A. Onwuanyi, K. Berekashvili and S. R. Bergmann, "Incidence of Major Cardiovascular Events in Black Patients with Normal Myocardial Stress Perfusion Study Results," Journal of Nuclear Cardiology, Vol. 8, No. 5, 2001, pp. 541-547. doi: $10.1067 / \mathrm{mnc} .2001 .116497$

[10] L. J. Shaw, R. C. Hendel, M. Cerquiera, J. H. Mieres, N. Alazraki, E. Krawczynska, S. Borges-Neto, J. Maddahi and C. N. B. Merz, "Ethnic Differences in the Prognostic Value of Stress Technetium-99m Tetrofosmin Gated Single-Photon Emission Computed Tomography Myocardial Perfusion Imaging," Journal of the American College Cardiology, Vol. 45, No. 9, 2005, pp. 1494-1504. doi:10.1016/j.jacc.2005.01.036

[11] A. Alkeylani, D. D. Miller, L. J. Shaw, M. I. Travin, H. G. Stratmann, R. Jenkins and G. V. Heller, "Influence of
Race on the Prediction of Cardiac Events with Stress Technetium-99m Sestamibi Tomographic Imaging in Patients with Stable Angina Pectoris," American Journal of Cardiology, Vol. 81, No. 3, 1998, pp. 293-297. doi:10.1016/S0002-9149(97)00896-5

[12] P. Poirier, T. D. Giles, G. A. Bray, Y. L. Hong, J. S. Stern, F. X. Pi-Sunyer and R. H. Eckel, "Obesity and Cardiovascular Disease: Pathophysiology, Evaluation, and Effect of Weight Loss: An Update of the 1997 American Heart Association Scientific Statement on Obesity and Heart Disease from the Obesity Committee of the Council on Nutrition, Physical Activity, and Metabolism," Circulation, Vol. 113, 2006, pp. 898-918. doi:10.1161/CIRCULATIONAHA.106.171016

[13] R. M. Krauss, M. Winston, B. J. Fletcher and S. M. Grundy, "Obesity: Impact of Cardiovascular Disease," Circulation, Vol. 98, 1998, pp. 1472-1476.

[14] R. H. Eckel, "Obesity and Heart Disease: A Statement for Healthcare Professionals from the Nutrition Committee, American Heart Association," Circulation, Vol. 96, 1997, pp. 3248-3250.

[15] J. E. Manson, W. C. Willett, M. J. Stampfer, G. A. Colditz, D. J. Hunter, S. E. Hankinson, C. H. Hennekens and F. E. Speizer, "Body Weight and Mortality among Women," The New England Journal of Medicine, Vol. 333, 1995, pp. 677-685. doi:10.1056/NEJM199509143331101

[16] E. E. Calle, M. J. Thun, J. M. Petrelli, C. Rodriguez, C. W. Heath Jr., "Body-Mass Index and Mortality in a Prospective Cohort of U.S. Adults," The New England Journal of Medicine, Vol. 341, 1999, pp. 1097-1105. doi:10.1056/NEJM199910073411501

[17] S. J. Hu, S. X. Liu, H. A. Katus and M. Luedde, ”The Value of Contrast Dobutamine Stress Echocardiography in Detecting Coronary Artery Disease in Overweight and Obese Patients," Canadian Journal of Cardiology, Vol. 23, No. 11, 2007, pp. 885-889. doi:10.1016/S0828-282X(07)70844-9

[18] S. G. Sawada, S. Sayyed, A. Raiesdana, I. Gradus-Pizlo, J. Mahenthiran and H. Feigenbaum, "Clinical Assessment and Rest and Stress Echocardiography for Prediction of Long-Term Prognosis in African Americans with Known or Suspected Coronary Artery Disease," Echocardiography, Vol. 26, No. 5, 2009, pp. 558-566. doi:10.1111/j.1540-8175.2008.00845.x

[19] K. Steel, R. Broderick, V. Gandla, E. Larose, F. Resnic, M. Jerosch-Herold, K. A. Brown and R. Y. Kwong, "Complementary Prognostic Values of Stress Myocardial Perfusion and Late Gadolinium Enhancement Imaging by Cardiac Magnetic Resonance in Patients with Known or Suspected Coronary Artery Disease," Circulation, Vol. 120, 2009, pp. 1390-1400. doi:10.1161/CIRCULATIONAHA.108.812503

[20] D. Poldermans, P. M. Fioretti, E. Boersma, J. J. Bax, I. R. Thomson, J. R. T. C. Roelandt and M. L. Simoons, "Long-Term Prognostic Value of Dobutamine-Atropine Stress Echocardiography in 1737 Patients with Known or Suspected Coronary Artery Disease: A Single-Center Experience," Circulation, Vol. 99, 1999, pp. 757-762.

[21] S. B. Labib, M. Goldstein, P. M. Kinnunen and E. C. 
Schick, "Cardiac Events in Patients with Negative Maximal versus Negative Submaximal Dobutamine Echocardiograms Undergoing Noncardiac Surgery: Importance of Resting Wall Motion Abnormalities," Journal of the American College Cardiology, Vol. 44, No. 1, 2004, pp. 82-87. doi:10.1016/j.jacc.2004.03.049

[22] R. Y. Kwong, H. Sattar, H. Wu, G. Vorobiof, V. Gandla, K. Steel, S. Siu and K. A. Brown, "Incidence and Prognostic Implication of Unrecognized Myocardial Scar Characterized by Cardiac Magnetic Resonance in Diabetic Patients without Clinical Evidence of Myocardial Infarction," Circulation, Vol. 118, 2008, pp. 1011-1020. doi:10.1161/CIRCULATIONAHA.107.727826
[23] L. Cortigiani, E. Picano, C. Coletta, F. Chiarella, W. Mathias, N. Gandolfo, M. De Alcantara, V. Mazzoni, G. F. Gensini and P. Landi, "Safety, Feasibility, and Prognostic Implications of Pharmacologic Stress Echocardiography in 1482 Patients Evaluated in an Ambulatory Setting," American Heart Journal, Vol. 141, No. 4, 2001, pp. 621629. doi:10.1067/mhj.2001.113997

[24] S. Ciaroni, A. Bloch and L. Albrecht, "Prognostic Value of Dobutamine Echocardiography in Elderly Patients with Suspected Coronary Artery Disease and an Abnormal Resting Electrocardiogram," Echocardiography, Vol. 19, No. 4, 2002, pp. 307-312. doi:10.1046/j.1540-8175.2002.00307.x 